La Revue

des Droits

de l'Homme

\section{La Revue des droits de l'homme}

Revue du Centre de recherches et d'études sur les droits fondamentaux

$17 \mid 2020$

Revue des droits de l'homme - $\mathrm{N}^{\circ} 17$

\title{
Les droits de la nature, des droits sans l'homme? Quelques observations sur des emprunts au langage du constitutionnalisme
}

\section{Manon Altwegg-Boussac}

\section{Q OpenEdition}

\section{Journals}

Édition électronique

URL : http://journals.openedition.org/revdh/8321

DOI : $10.4000 /$ revdh.8321

ISSN : 2264-119X

Éditeur

Centre de recherches et d'études sur les droits fondamentaux

Référence électronique

Manon Altwegg-Boussac, "Les droits de la nature, des droits sans l'homme ? Quelques observations sur des emprunts au langage du constitutionnalisme », La Revue des droits de l'homme [En ligne], 17 | 2020, mis en ligne le 30 janvier 2020, consulté le 19 octobre 2020. URL : http:// journals.openedition.org/revdh/8321 ; DOI : https://doi.org/10.4000/revdh.8321

Ce document a été généré automatiquement le 19 octobre 2020.

Tous droits réservés 


\title{
Les droits de la nature, des droits sans l'homme? Quelques observations sur des emprunts au langage du constitutionnalisme
}

\author{
Manon Altwegg-Boussac
}

1 Des droits naturels aux droits de la nature, la philosophie des droits de l'homme s'oriente vers des chemins escarpés ${ }^{1}$. Ce n'est plus l'homme sujet de droits naturels qu'il s'agit de considérer mais la nature, entendue dans sa diversité d'« éléments » - au sens large: animaux, fleuves, montagnes, espaces naturels, paysages, végétaux et minéraux - sujet de droits contre un anthropomorphisme délétère ${ }^{2}$. La stratégie rhétorique n'a rien de nouveau : utiliser un langage juridique classique bien ancré pour porter des combats nouveaux. Les mêmes concepts, catégories juridiques et modes de justifications sont donc à l'œuvre, ceux hérités du constitutionnalisme et des droits de l'homme, mais l'équilibre général tout comme les fins ont changé. A la manière dont on tente ailleurs de penser la constitution sans l'Etat, ou la démocratie sans le peuple, on envisage ici de penser les droits sans l'homme.

Le glissement de concepts que suggère la reconnaissance des droits de la nature soulève donc des craintes légitimes. La récupération de concepts pour leur connotation positive est à la fois contestable sur le plan scientifique - on change de contenu sans le dire- et préoccupant sur le terrain politique - qu'en sera-t-il du rapport au pouvoir? Qui représentera la nature ? Comment le tout organique peut prévaloir sur l'individualisme libéral ? Tout l'intérêt de la doctrine constitutionnelle est de pointer ces glissements, parfois silencieux, particulièrement lorsqu'ils pourraient avoir des conséquences directes sur la manière d'envisager le pouvoir; il n'est pas anodin à cet égard de constater que le discours des droits de la nature sert parfois un discours autoritaire ${ }^{3}$. Au-delà de ces difficultés, les discours relatifs aux droits de la nature recèlent aussi quelques attraits. Comprendre les logiques qui sont à l'œuvre invite à réfléchir à l'adéquation et à l'utilité concrète de nos catégories juridiques classiques pour porter 
des revendications nouvelles, et notamment en matière environnementale. Après tout, les droits de l'homme contiennent une charge "combattive " ${ }^{4}$ destinée à les adapter aux besoins des époques.

3 Tous les partisans des droits de la nature partagent une critique virulente de l'anthropocène, terme assez médiatisé ${ }^{5}$, qui cristallise une ère de domination des hommes vis-à-vis de leur environnement naturel. De ce point de vue, les apports du droit international de l'environnement semblent pris dans le même piège: en renforçant les obligations à l'égard des individus et des entreprises, ils ne feraient que maintenir ce biais anthropocentrét. Avec les droits de la nature, il s'agit moins d'insister sur le contenu des obligations juridiques - elles sont déjà existantes dans le droit $^{7}$ - mais plutôt sur la manière de les dire: considérer les droits de certains éléments de la nature produirait, par ce déplacement du discours, davantage d'effet sur les consciences politiques et sociales et donc sur notre perception du monde. Beaucoup d'espoirs sont ainsi placés dans des mots ${ }^{8}$ face à ce qui est présenté comme une " urgence morale $»^{9}$. La transposition des catégories juridiques des droits de l'homme serait donc une manière, presque psycho-sociale, de modifier nos représentations du monde ${ }^{10}$ et de prendre au sérieux le pavé jeté dans la mare par Christopher Stone en $1972 »$ Should trees have standing? ${ }^{11}$.

y soit ou non favorable, les constitutionnalistes sont invités à s'y intéresser. En effet, les discours des droits de la nature ont déjà largement emprunté le vecteur du droit constitutionnel et politique en se plaçant à trois niveaux différents. Le premier type de discours relève de l'éthique environnementale tout en touchant parfois la théorie constitutionnelle et politique. On y trouve des réflexions relatives aux fondements de légitimité du pouvoir au sein d'une démocratie libérale, des critiques plus ou moins sérieuses du contrat social, du droit naturel moderne et de l'individualisme ${ }^{12}$. Le deuxième type de discours se situe sur un terrain conceptuel assumé. Les concepts sont mobilisés de manière à les adapter aux fins recherchées. Tout comme la personnalité morale a été le fruit d'une construction conceptuelle, la reconnaissance des droits de la nature suppose un effort similaire pour repenser les concepts classiques de sujet, de personnalité juridique ${ }^{13}$, de droit ${ }^{14}$, de représentation ${ }^{15}$ de responsabilité etc. Ils sont de ce point de vue soumis à la critique au regard de leur intérêt et de leur opérabilité. Un dernier type de discours relève plus prosaïquement du droit constitutionnel positif. Il est composé d'arguments divers, plus ou moins convaincants, sans nécessairement être accompagné d'une théorie des droits de la nature ou d'une philosophie politique définie.

5 Au cours des dix dernières années, fleuves, forêts, glaciers, parcs et ensembles naturels se sont vus reconnaître des droits dans plusieurs systèmes juridiques. Les droits de la nature ont été formalisés dans le Chapitre $7 \mathrm{de}$ la Constitution de l'Equateur, pionnière en la matière ${ }^{16}$. La Charte Bolivienne, la Loi sur les droits de la Terre Mère, fut ensuite adoptée en 2010avec un fort retentissement international. Elle énonce dans son article 5 que la Pacha Mama est un "sujet collectif d'intérêt général ", titulaire de droits ${ }^{17}$. Par deux lois du Parlement de la Nouvelle-Zélande, la personnalité juridique sera accordée en 2014 au parc naturel Te Urewera puis en 2017 au fleuve Whanganui ${ }^{18}$. En Australie, l'Etat de Victoria a construit un dispositif moins décisif mais qui emprunte indirectement le chemin de la reconnaissance des droits de la nature ${ }^{19}$. Enfin, après le précédent équatorien évoquant une "démocratie de la Terre ${ }^{20}$, les jurisprudences colombienne et indienne ont récemment reconnu des droits aux fleuves. En Inde, la 
Haute Cour de l'Etat Uttarakhand par deux jugements rendus le 20 puis le 30 mars 2017, reconnaît la personnalité juridique aux deux fleuves, le Gange et la Yamuna, puis dans un second jugement, aux ensembles naturels les englobant, et à deux glaciers au sein desquels ils prennent leur source ${ }^{21}$. La Cour affirme qu' « au-delà de nos devoirs juridiques et constitutionnels, c'est notre devoir moral de protéger l'environnement et l'écologie $»^{22}$. En Colombie, dans un jugement de 2016, la Cour constitutionnelle a conféré la personnalité juridique au fleuve $A$ trato $^{23}$, tout en reprenant son concept déjà élaboré auparavant de " constitution écologique ».Elle opposeà l'anthropocentrisme de l'Ouest une approche «écocentrique » qui serait propre au constitutionnalisme colombien ${ }^{24}$.

Les droits de la nature sont ainsi colorés du langage du constitutionnalisme ${ }^{25}$. Ils puisent dans sa double dimension axiologique, à travers le langage de la garantie des droits ${ }^{26}$, et politique: les droits de la nature ne sont pas détachés de certaines perceptions du "peuple", générations futures, minorités ou peuples autochtones. La doctrine des droits fondamentaux avait déjà entamé cette évolution conceptuelle des droits de l'homme en réduisant le «sujet universel» à une "possibilité parmi d'autres ${ }^{27}$. Mais cet emprunt au constitutionnalisme fait naître une tension évidente : le libéralisme, la démocratie, les droits de l'homme s'accordent mal avec l'idée des droits de la nature ${ }^{28}$. Cette tension se traduit dans les différents discours ayant consacré des droits à des éléments naturels: l'argumentation déployée au soutien de cette reconnaissance semble tiraillée entre un langage des droits et un langage politique.

Ces deux types de registres argumentatifs sont présents dans les différents cas reconnaissant les droits de la nature. Certains arguments constitutionnels puisent dans le registre axiologique et affectent notamment la perception des droits, considérés comme «droit naturels ». D'autres arguments empruntent plutôt le registre politique du constitutionnalisme sans être totalement détachés des premiers.

$8 \quad$ I Le registre axiologique : Les droits de la nature, des droits naturels ?

9 II Le registre politique : Des droits pour les peuples, les minorités et les générations futures?

\section{Les droits de la nature, des droits naturels ?}

La reconnaissance des droits de la nature repose sur la perception axiologique du constitutionnalisme. On retrouve ainsi les ressorts du droit naturel sans pourtant que cette conception soit pleinement déployée. Elle contient une potentielle difficulté " anti-moderne ${ }^{29}$ en fragilisant l'individualisme libéral au fondement de nos sociétés. L'argumentation en faveur des droits de la nature présente donc une certaine ambivalence: celle-ci oscille entre une approche naturaliste (ou essentialiste) d'une part, et selon laquelle les droits de la nature sont inhérents à la nature elle-même, et une approche conventionnaliste (appelée aussi «technique ") ${ }^{30} \mathrm{~d}$ 'autre part, selon laquelle les droits de la nature se présentent comme un moyen juridique en vue d'assurer une protection efficace de la nature.

\section{D'un droit naturel de la nature}

11 La reconnaissance des droits de la nature se sert des ressorts du droit naturel. Il ne s'agit plus du droit naturel moderne : le droit naturel dont il est question dépasse la 
«nature humaine» pour englober le «vivant» (la biodiversité) et plus largement encore, la " nature ", entendue sous l'angle d'un écosystème comprenant une diversité d'éléments, animés ou non, et tissant des relations interdépendantes. Ces présupposés naturalistes ne sont pas clairement exprimés. La tentation est forte de se servir d'un piège de langage en jouant sur le terme de "nature ». Celui-ci peut revêtir une connotation matérielle, les éléments de la nature, ou une connotation essentialiste, renvoyant à un ordre préexistant de valeurs. Avec cette ambivalence, plus ou moins recherchée, il n'est presque plus besoin d'éclairer ces présupposés naturalistes : le mot même de la « nature » semble littéralement en injecter l'idée.

L'approche de droit naturel repose sur une logique bien connue qui est simplement adaptée à l'enjeu des droits de la nature. Elle conduit à observer des "valeurs » considérées comme "intrinsèques » - cet adjectif, devenu commun, est présent dans les cas concrets équatorien, colombien, néo-zélandais, et indien - parfois même présentées comme "sacrées» (la Pacha Mama, ou le cas indien) ${ }^{31}$.Ces valeurs préexistantes imposent dans la logique du droit naturel le respect de principes éthiques et moraux qui devraient être réceptionnés par le droit positif. Dans les cas reconnaissant les droits de la nature, cette "réception »se traduit essentiellement par le passage de la considération du "vivant " comme valeur préexistante, à celui de la reconnaissance par le droit positif d'un "droit». Ainsi l'article 71 de la Constitution équatorienne est formulé de la manière suivante: "La Nature ou Pacha Mama, où se reproduit et se réalise la vie, a le droit à ce que l'on respecte intégralement son existence et le maintien et régénération de ses cycles vitaux, sa structure, ses fonctions et ses processus

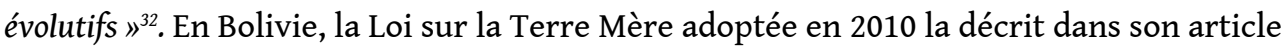
3 comme un "système dynamique vivant " comprenant une "communauté indivisible" d'organismes et systèmes vivants "interdépendants et complémentaires", et qui " partagent une destinée commune». Le premier droit qui est consacré est le droit à la vie qui renvoie au «droit à la perpétuation de l'intégrité des écosystèmes et des processus naturels qui les soutiennent ». Le langage des droits naturels privilégie la référence au « vivant » : la jurisprudence indienne affirme ainsi que « les rivières, les forêts, les lacs, les étendues d'eau, l'air, les glaciers et les sources ont un droit à l'existence [...]et un droit à régénérer leur propre système écologique vital. Les rivières ne sont pas seulement des étendues d'eau. Elles sont scientifiquement et biologiquement vivante». La Haute Cour poursuit en conférant un «droit constitutionnel à la Terre Mère " "3 $^{33}$ Le passage du «vivant », entendu comme valeur en soi, à l'attribution d'un « droit » apparaît ainsi presque évident.

Dans cette perspective, la seule appartenance à l'écosystème suffirait à la revendication d'un droit. Mais comment connaître ces hiérarchies de valeurs entre les hommes et les animaux, entre les animaux et les plantes entre les plantes et l'air? Quelle institution pourra définir ces "valeurs intrinsèques $»^{34}$ ? Quelle place sera donnée à la science et comment passera-t-on de la proposition scientifique à la valeur morale ? Au-delà de ces difficultés, propres à toute conception naturaliste, une contradiction insoluble apparait: une approche naturaliste fondée sur la seule considération du vivant pourrait conduire à renoncer au primat de l'individu. Les droits de la nature rompraient avec les fondements du constitutionnalisme moderne tout en utilisant son langage. Les différentes argumentations en faveur des droits de la nature ne s'embarrassent pas de ces questions : les institutions puisent dans les ressources leur permettant de justifier cette consécration. Tout en versant dans le naturalisme ${ }^{35}$, les 
droits de la nature se présentent aussi comme un moyen juridique de protéger efficacement la nature.

\section{Des droits pour protéger la nature}

14 Cette autre approche, dite "conventionnaliste", conduit à envisager les catégories juridiques comme des moyens de changer notre rapport à la nature. L'exemple indien en constitue une bonne illustration. La Haute Cour déclare que l'ensemble naturel considéré contient des droits et devoirs d'une personne vivante "dans le but de la préserver et la conserver $\rrbracket^{36}$. La Cour insiste également, dans les deux jugements, sur la dimension fictive de «personne juridique » qui a été créée pour répondre aux «besoins d'une société $\rrbracket^{37}$. Elle affirme ainsi que la personnalité juridique peut bien être conférée à des éléments vivants ou non, à des objets ou à des choses, du moment que cette reconnaissance est justifiée par les attentes d'une société dans un contexte environnemental menacé ${ }^{38}$. En outre, la consécration de la personnalité juridique s'accompagne dans les cas néo-zélandais, colombien et indien de structures de représentation façonnées par le droit pour administrer ses intérêts ${ }^{39}$.

Avec ce rapport moyen/fin les difficultés précédentes sont évitées oudu moins, elles ne se posent plus de la même manière. Il ne s'agit plus de savoir si les végétaux et les animaux ont en soi la même "valeur» que les hommes, mais comment découper le vivant d'une manière qui corresponde aux attentes et aux conceptions du monde, entre des perceptions holiste et individualiste. Ces choix conceptuels doivent donc être justifiés et argumentés, en ayant par exemple recours à des données scientifiques ou à la philosophie morale. La discussion est dense. Certains critères tirés de la " sensibilité " ou des "intérêts " permettent de ne retenir comme "valeur morale " que les droits des animaux et des humains; d'autres sont focalisés sur le «besoin » ou le « vivant » de manière à englober, au même niveau, tous les organismes vivants, en excluant les éléments «non biotiques » qui n'auraient qu'une valeur « instrumentale » à défaut d'être «intrinsèque $»^{40}$; enfin une prise en compte globale de l'écosystème, incluant aussi les micro-organisme et éléments inanimés, conduirait à une reconnaissance très générale de ces droits.

16 Il est donc tout à fait possible de construire des concepts juridiques de manière à faire prévaloir les intérêts des hommes sur les autres ${ }^{41}$. Les droits de l'homme peuvent bien se maintenir sans craindre d'être réduit à des éléments parmi d'autres: il suffit d'adapter les montages juridiques ${ }^{42}$. En outre, les « droits » dont il est question dans ces divers cas concrets ne sont pas les mêmes que les droits de l'homme: certains sont spécifiques aux droits de la nature (droit à la restauration etc...), d'autres sont communs, le droit d'agir en justice, la liberté contractuelle, ou le droit de propriété, et d'autres sont exclus : les droits politiques ${ }^{43}$. Ces derniers ne sont pas envisagés - du moins en droit positif car l'intérêt intellectuel demeure ${ }^{44}$ - les hommes peuvent donc dormir tranquille, dans leur trou de verdure, il n'y aura point d'arbres au Parlement, pour l'instant du moins. 


\section{Les droits de la nature, des droits pour le(s) peuple(s), les minorités et les générations futures ?}

17 A chaque fois que les droits de la nature sont consacrés, le lien politique n'est jamais loin. Il apparait à travers la prise en compte des "peuples autochtones» ou des « minorités »ayant tissé des liens particuliers avec les éléments de la nature, ou encore par le biais des» générations futures ». La question des droits de la nature ne repose donc pas seulement sur le langage de la garantie des droits, elle contient aussi une dimension politique à travers la référence même indirecte $\mathrm{au}(\mathrm{x})$ peuple(s). Cette ressource politique constitue un moyen utile de justifier la consécration de tels droits sur un terrain, cette fois, classique du constitutionnalisme (et donc stratégiquement plus convaincant que ne l'est le recours à un droit naturel de la nature). Pourtant, son poids demeure incertain. Au point que l'on peut se demander si la reconnaissance des droits de la nature ne conduit pas aussi à privilégier le langage des droits sur le langage politique.

La référence $\mathrm{au}(\mathrm{x})$ « peuple(s) » apparait à la fois dans l'argument, peu approfondi, des générations futures, et à travers l'argument pluraliste : un peuple comprenant diverses communautés, dans un contexte historique marqué par le colonialisme.

\section{Les générations futures}

19 La prise en compte des générations futures est, avec la valeur intrinsèque, une référence régulière des discours juridiques tournés vers la protection de l'environnement. La Déclaration de Rio sur la biodiversité en 1992 affirme la détermination des Etats à "conserver et à utiliser durablement la diversité biologique au profit des générations présentes et futures ». L'article 33 de la Constitution bolivienne énonce ainsi : "l'exercice de ce droit est de permettre aux individus et communautés des générations présentes et futures, ainsi que les autres êtres vivants, de se développer normalement et de façon permanente ». La constitution équatorienne tout comme les jurisprudences indienne et colombienne la reprennent aussi ${ }^{45}$.

20 Cette référence aux générations futures rappelle la littérature du constitutionnalisme du XVIIIe siècle. Dans la perspective jeffersonienne, tout comme chez Condorcet et Paine, la constitution ne devait pas se transformer en une « dead hand» conduisant à soumettre les générations futures à un peuple mort, le peuple constituant. L'attention des constitutionnalistes du XVIIIe siècle pour ces générations exprime la difficulté, bien connue, à concilier d'un côté l'expression politique du peuple vivant (chaque génération doit être en mesure de décider de son avenir constitutionnel) et d'un autre côté la permanence de l'œuvre constitutionnelle, comprise comme un facteur de paix sociale, contre son altération (et l'on retrouve la distinction de Sieyès entre pouvoir constituant et pouvoirs constitués).Cette réflexion en termes de générations a plutôt disparu de la doctrine constitutionnelle contemporaine mais elle apparait, sous un autre jour, dans le discours des droits de la nature.

21 La référence aux générations futures figure dans les différents cas concrets sans être vraiment approfondie. C'est la doctrine qui l'investit davantage. Tout comme l'idée constitutionnelle, la protection de la nature apprivoise une temporalité particulière du "peuple». Alors que chez Jefferson "The earth belongs always to the living generation", 
les droits de la nature, dans la mesure où la survie des hommes dépend de cet environnement écologique, garantissent, eux, l'expression d'un "peuple constituant intergénérationnel $»^{46}$. La volonté politique qu'il s'agit de préserver n'est donc plus celle de la nation abstraite, ni d'une humanité transcendante, c'est celle d'un peuple pris dans son environnement naturel, d'une ère des humains, comme donnée "scientifico-biologique", et dont l'existence est menacée sans une forte prise de conscience politique ${ }^{47}$. Dans cette perspective, les droits de la nature sont présentés comme une condition de la démocratie, plus importants encore que les droits politiques, puisqu'ils en conditionnent l'exercice par-delà les générations ${ }^{48}$. La difficulté centrale du constitutionnalisme rejaillit pourtant avec un écho familier : et si le peuple n'a que faire de sa destruction? Comment pourrait-on légitimement le forcer à être vivant, pour être libre?

\section{Les populations autochtones}

Les droits de la nature sont également systématiquement reliés à la présence de populations riveraines, autochtones, qui revendiquent d'autres conceptions du monde et de la nature. Il s'agit cette fois de prendre en compte un peuple dit pluraliste ou multiculturel, composé des groupes, minorités, communautés, populations autochtones, et qui serait le résultat de combats historiques et politiques menés sous fond d'autoritarisme et de colonialisme. Ce n'est donc pas un hasard si les discours des droits de la nature sont nés dans des systèmes juridiques où la colonisation a été éprouvée par ces mêmes populations ${ }^{49}$.

Ce lien entre droits de la nature et "populations autochtones" ou "minorités » demeure pourtant incertain: les droits de la nature existent-ils en vertu des cosmovisions de ces populations autochtones ou s'agit-il de défendre les droits de la nature indépendamment d'elles? La balance entre droits de la nature et reconnaissance politique des communautés est instable. Les cas équatorien et néozélandais privilégient cette dimension politique quand les exemples colombien et indien, tendent à lui préférer le langage des droits. Cette distinction est évidemment liée au support juridique de cette reconnaissance et dépend largement des différentes cultures constitutionnelles. Dans le premier cas, les droits sont consacrés par un acte intrinsèquement politique (la nouvelle constitution équatorienne et la loi du Parlement néo-zélandais); alors que dans le second c'est la jurisprudence (indienne et colombienne) qui y procède, la dimension politique s'y trouve dès lors davantage entremêlée, voire confondue, avec le langage familier des droits.

Les cas équatorien et néo-zélandais. Dans les cas équatorien et néo-zélandais, la consécration des droits de la nature est reliée à l'expression d'une volonté politique, affirmée à un niveau constitutionnel (formel ou politique). Ainsi, le nouveau constitutionnalisme latino-américain, né dans les années 2010, adopte une conception multiculturelle de la nation, en mesure d'accueillir les droits de la nature ${ }^{50}$. Ce mouvement se caractérise notamment par une volonté de rupture avec un passé autoritaire et par l'affirmation d'une approche dite « inclusive », intégrant l'expression politique des groupes et minorités. Le Préambule de la Constitution de l'Equateur est éloquent :» Nous, peuple souverain de l'Équateur, reconnaissant nos racines millénaires, forgées par des femmes et des hommes de différents peuples, célébrant la nature, la Pacha Mama, dont nous faisons partie et qui est vitale pour notre existence, invoquant le nom de Dieu et en 
reconnaissant nos diverses formes de religiosité et de spiritualité, s'appuyant sur la sagesse de toutes les cultures qui nous enrichissent en tant que société, en tant qu'héritiers des luttes sociales de libération contre toutes les formes de domination et de colonialisme, et avec un engagement profond pour le présent et l'avenir, Nous décidons de construire une nouvelle forme de vie citoyenne en commun, dans la diversité et l'harmonie avec la nature, pour atteindre le bien vivre, le sumak kawsay $\aleph^{51}$. La Constitution équatorienne réceptionne donc les droits de la nature tout en les rattachant aux cultures et à la cosmologie amérindienne dont le Sumak Kawsay, qui renvoie au «bon vivre» des peuples, et la Pacha Mama, sont une expression directe ${ }^{52}$.

D'une manière similaire, mais dans une culture constitutionnelle différente, les deux lois adoptées par le Parlement de Nouvelle-Zélande, en 2014 pour le parc Te Urewera, et en 2017 pour le fleuve Whanganui, sont la concrétisation d'accords politiques passés entre le gouvernement et les populations autochtones riveraines ${ }^{53}$. Ces dernières revendiquaient leurs droits ancestraux contre la gestion arbitraire de la propriété par les colons britanniques. La loi de 2014 vient consacrer la personnalité juridique de l'ensemble naturel Te Urewerea tout en reconnaissant sa valeur sacrée et spirituelle pour la population Tuhoe. Elle insiste sur le maintien de cette connexion entre les Tuhoé et le parc naturel, et sur la nécessité de sa préservation en tant qu'héritage culturel et ressource spirituelle. Par la loi de 2017, le fleuve Whanganui est considéré dans un ensemble indivisible, comprenant des éléments " physiques et métaphysiques ". La loi fait donc référence aux concepts culturels et spirituels des Maoris ainsi qu'à la période historique de la colonisation britannique ${ }^{54}$. Ces deux lois viennent préserver un accord politique visant à réparer ces injustices et à reconnaître les conceptions du monde de ces populations autochtones.

Ainsi, dans ces deux cas équatorien et néo-zélandais, la reconnaissance des droits de la nature procède d'une volonté politique forte, celle d'intégrer les revendications de ces populations tout en reconnaissant la valeur culturelle de leurs cosmologies holistes ${ }^{55}$. Les conceptions du monde des populations autochtones et modernes seraient cette fois réunies au sein de ce corps politique multidimensionnel. Cette présentation d'un droit dit "pluraliste " ${ }^{56}$ souffre de quelques faiblesses. Que l'on se félicite de la "capacité grandissante du droit moderne à façonner le vivre ensemble d'une société ${ }^{57}$, la réception des cosmovisions autochtones se fait dans le langage et par les outils du droit "moderne " 58. Par ailleurs, il n'est pas certain que les discours des droits de la nature demeurent pleinement rattachés à ces cosmologies autochtones. Dans le cas de l'Equateur, les travaux constituants montrent que tout en reconnaissant cet héritage culturel, les constituants se sont aussi réapproprié les droits de la nature, en les défendant de manière autonome, pour eux-mêmes, au nom d'une conception écocentrique du monde ${ }^{59}$. De manière plus incidente, la loi de 2014 de Nouvelle-Zélande contient un paragraphe intitulé "Te Urewera et tous les néo-zélandais» dans lequel la valeur intrinsèque du parc naturel est reconnue, au-delà des cosmologies autochtones, à l'égard des néo-zélandais ${ }^{60}$; de même le contenu de l'accord politique "Ruruku Whakatupua ", signé avec la Couronne en 2012 en amont l'adoption de la loi de 2017, consacre "la valeur naturelle, scénique et de conservation" de l'ensemble naturel englobant le fleuve Whanganui, sans la déduire des conceptions des Maoris ${ }^{61}$. Les cas néo-zélandais et équatorien révèlent donc aussi une réelle ambivalence. Elle se traduit par un certain détachement vis-à-vis des revendications des populations autochtones. Cette potentielle réappropriation des «droits de la nature " pourrait donc conduire à une autonomisation du langage des droits par rapport à la question de la 
reconnaissance de l'héritage culturel et celle de l'intégration politique des populations autochtones.

Les jurisprudences indienne et colombienne : la prévalence d'un langage des droits

expression culturelle et politique des peuples autochtones se traduit dans les jurisprudences indienne et colombienne de manière différente, sans faire référence à un volontarisme politique. Les jugements de la Haute Cour de l'Etat de l'Uttarakhand s'intéressent aux membres la communauté hindous en soulignant qu'ils sont» connectés » à ces rivières. C'est donc bien à travers leur perception que la Cour retient le caractère "sacré » des fleuves ${ }^{62}$. Mais cette prise en compte demeure très vague et sommaire (il est même suggéré que la Haute Cour ait voulu suivre l'impulsion donnée par le cas Néo-Zélandais rendu quelques jours avant); la Haute Cour insiste plus généralement sur les croyances de l'ensemble de la population indienne dont la moitié dépend de la ressource fluviale. Elle affirme ainsi que les fleuves du Gange et de la Yamuni constituent une ressource «à la fois matérielle et spirituelle »qui «nourrit les communautés de la montagne à la mer ». Elle fait aussi référence à la " foi » de la société. En respectant ces cultures et croyances, la Haute Cour semble présenter son argumentation comme une manière indirecte - et très évasive - de tisser un lien de confiance avec la société ${ }^{3}$.

Le jugement de la Cour constitutionnelle colombienne repose sur une autre logique, celle des droits «bioculturels $»^{64}$. Après avoir défendu une approche écocentrique de la constitution, considérée comme un "élément transversal de l'ordre constitutionnel colombien ${ }^{65}$, la Cour rattache cette conception de la nature au pluralisme culturel. Elle prend en compte, de manière beaucoup plus approfondie que ne le fait la Haute Cour indienne, la présence des communautés afro-colombiennes, riveraines du fleuve Atrato, en insistant sur l'importance spirituelle de ces ressources pour ces communautés ${ }^{66}$. La rhétorique des droits bioculturels ${ }^{67}$ conduit ici à fusionner le volet droit de la nature (protection des ressources naturelles) et le volet droit politique (protection de la culture des citoyens afro-colombiens) dans un même concept. Tel un concept moderne en miroir de la cosmologie de ces communautés, les droits bioculturels demeurent une construction encore hasardeuse ${ }^{68}$ : ces droits semblent dire tout mais ne disent rien. En effet la dimension politique, qui était bien présente dans le cas du réformisme constitutionnel équatorien ou des accords politico-constitutionnels néozélandais, semble ici diluée dans un langage des droits. Mais au sein même de l'équilibre interne de ces droits bioculturels, le volet nature pourrait l'emporter sur le volet culture : cela se manifeste notamment par une doctrine ambigüe relative à l'existence d'un » devoir d'intendance » de la nature dont les populations autochtones seraient débitrices ${ }^{69}$. Il ne s'agirait alors même plus de protéger le droit culturel mais de privilégier le droit de la nature par le truchement du savoir des populations considérées. Le risque est celui d'une « dépolitisation ${ }^{70}$ des droits des populations autochtones reniant du même coup des siècles de combat politique.

La prise en compte des droits de la nature fait face à une double hésitation. D'un côté, les arguments juridiques ont tendance à sombrer dans un naturalisme déshumanisé, d'un autre côté, ils demeurent reliés à la reconnaissance des cosmologies des populations autochtones dans un contexte postcolonial. C'est en effet dans ces contextes et ces cultures locales que la question écologique a trouvé un levier politique. Les initiatives récentes, néo-calédonienne et corse, ouvrent d'ailleurs la voie à de telles évolutions, qu'il s'agisse de la reconnaissance de la personnalité juridique à certains 
éléments de la nature, selon la conception de la vie de la société kanak $^{71}$, ou de la perspective d'adoption d'une Charte de la nature spécifique à la collectivité de Corse ${ }^{72}$. L'enjeu « politique » demeure au final profondément lié au « local » et la rhétorique des droits de la nature peine à s'en désolidariser.

\section{NOTES}

1. Cet article est issu du colloque organisé par Olivier Clerc à l'Université de Corse-Pascal-Paoli, Ethniques naturalistes et droits de l'environnement: d'une révolution conceptuelle à une refondation juridique? septembre 2018

2. E. Millard, "Anthropocène: Des droits naturels aux droits de la nature", in Résilience et résistance,Des Pactes internationaux des droits de l'homme à l'épreuve d'une société internationale postmoderne, dir. S. Grosbon, Paris, Pedone, 2018, p. 89 et s

3. J. Wayne Smith, D. Shearman, The Climate Change Challenge and the Failure of Democracy, Praeger, 2007, 208 p. Voir aussi sur l' « éco-autoritarisme »: R. Eckersley, «Liberal democracy and the rights of nature: The struggle for inclusion", in Ecology and democracy, in Environmental Politics · December 1995p. 169-198 etP. Brunet, «Vouloir pour la nature. La représentation juridique des entités naturelles ", in Journal of interdiscipliinary history of ideas, Volume 8 section 2, 2019, p. 2.6.

4. Comme l'écrit Danièle Lochak, «L'arme juridique doit donc être combinée avec l'arme politique et utilisée à l'appui des combats politiques, non comme une alternative à ces combats. Telle est la condition pour qu'on puisse parler d'usages « militants » du droit. ». Danièle Lochak, "Les usages militants du droit». La Revue des Droits de l'Homme, CTAD-CREDOF (Centre de recherche et d'études sur les droits fondamentaux) 2016, ff10.4000/revdh.2178ff. ffhal01647284f. Elle est favorable au maintient de l'expression droits de l'homme: " Mais ce qui retient plus encore d'abandonner les «droits de l'homme» au profit des «droits humains ", c'est au les premiers renvoient à une tradition, à des idéaux, à des combats politique. C'est cette dimension là, de l'ordre de la mémoire de l'histoire, qu'on risquerait de laisser échapper en renonçant à utiliser l'expression » p. 11. D. Lochak, Les droits de l'homme, $3^{\mathrm{e}}$ éd. , Repères, 2009, p. 4 et s.

5. Ce terme est aussi critiqué pour son imprécision. Selon Eric Millard « Parler « d'anthropocène » ici sacrifie vraisemblablement à un phénomène de mode. Mais, le terme est désormais mobilisé sans véritable justification, de manière très métaphorique, dans certaines sciences sociales, pour désigner la période qu'on considère traditionnellement comme celle de l'humanité, c'est-à-dire l'époque de l'histoire de notre monde spatio-temporel durant laquelle l'existence même de l'humanité, et les activités que celle-ci développe, a des conséquences sur le milieu ambiant ». E. Millard, « Anthropocène... », préc., p. 89.

6. E.L. O'Donnell and J. Talbot-Jones, “Creating Legal Rights for Rivers: Lessons from Australia, New Zealand, and India", in Ecology and Society, vol. 23, n. 1, 2018, p. 7 : "However, the counter to this argument is that these other advances in environmental law actually provide two powerful reasons for extending legal rights to nature. First, from a philosophical perspective continuing to prosecute environmental cases on the basis of ever-more attenuated "harm" to humans relies on an increasingly convoluted and anthropocentric argument, which obscures the needs of nature."

7. Concernant la question de la prise en compte du droit de l'environnement dans le contentieux constitutionnel français voir notamment : P. Rrapi, « Le Conseil constitutionnel (aussi) voit les 
glaciers fondre, À propos de la décision du 11 octobre 2019 du Conseil constitutionnel (2019-808 QPC), Société Total raffinage », Actualité Droits-Libertés, Décembre 2019, disponible : https:// doi.org/10.4000/revdh.7583.

8. Comme l'écrit P. Brunet « la reconnaissance de droits à la nature semble motivée par le besoin désespéré de mettre fin à la destruction écologique. Mais à quoi cela sert-il si le droit est indéfini au point de n'être qu'une coquille vide queremplissent ceux qui l'appliquent ?». P. Brunet, « Les Droits de la nature et la personnalité juridique des entités naturelles en Nouvelle-Zélande: un commun qui s'ignore? » in, Storia costituzionale n. 38, II semestre, 2019, pp. 39-53, p. 41.

9. J.A. Nash, "The Case for Biotic Rights", in Yale Journal of International Law, vol. 18, n. 1, 1993, pp. 235-250, p. 248

10. D.R. Boyd, The Rights of Nature : A Legal Revolution that Could Save the World, Toronto,ECW Press, 2017.

11. C. Stone, Should Trees Have Standing ? Toward Legal Rights for Natural Objects, in «Southern California Law Review», vol. 45, 1972. Voir aussi M.-A. Hermitte , " Le concept de diversité biologique et la création d'un statut de la nature ». in L'Homme, la nature et le droit, B. Edelman et M.-A. Hermitte, Paris, Christian Bourgois, p. 238, 2011. Id., " La nature, sujet de droit ? " Annales. Histoire, Sciences Sociales, vol. 66, 1, p. 173-212.

12. Ethique de l'environnement, Nature, valeur, respect, Texte réunis par H. S. Afeissa, Vrin, 2007. Et notamment R. Sylvain (Routley), «A-t-on besoin d'une nouvelle éthique, d'une éthique environnementale?», p. 31; B Norton, "L'éthique environnementale et l'anthropocentrisme faible», p. 249 ; C. Stone, «Le pluralisme moral et le développement de l'éthique environnementale », p. 285.

13. M. A. Hermitte, « La nature, sujet de droit ?», préc.; id. « Artificialisation de la nature et droit du vivant », P Descola (dir.), Odile Jacob, Collègue de France, 2018, p. 257-284.

14. Par exemple, J. Nedelsky, J. Reconceiving Rights as Relationship, Review of Constitutional Studies / Revue d'études constitutionnelles, Volume 1, Issue 1 (1993).Elle envisage les droits comme des « relations évolutives " plutôt que des « droits individuels » fixes et immuables.

15. Voir sur la question de la représentation les contributions de Pierre Brunet: P. Brunet, « Les Droits de la nature et la personnalité juridique des entités naturelles en Nouvelle-Zélande: un commun qui s'ignore?», préc.; id., «Vouloir pour la nature. La représentation juridique des entités naturelles »,préc.; id., «L'écologie des juges - la personnalité juridique des entités naturelles en Inde et en Colombie ", à paraitre chez Mare et Martin, 2020 (la notification de la page sera ajoutée dès que possible).

16. Constitution de l'Equateur (2008), Titre 2, Chapitre 7, Droits de la nature, art. 71 à 74.

17. Loi du 21 décembre 2010, (Loi $n^{\circ} 71$ ), Loi sur les droits de la terre Mère. Son article 7 dispose que la Terre Mère a droit à la vie (le maintien de son intégrité), à la diversité (c'est-à-dire à ne pas être uniformisée par modification génétique), à l'accès à l'eau, à l'air pur, à l'équilibre, à la restauration et à une existence sans pollution. Une autre loi sera adoptée le 15 octobre 2012 (Loi $\mathrm{n}^{\circ} 300$ ) Loi-cadre de la Terre Mère et du développement du Bien vivre.

18. Te Urewara Act 2014 . L'article 14, conformément à l'engagement pris en 2012, dispose : « Te Awa Tupua declared to be legal person" Te Awa Tupua (Whanganui River Claims Settlement) Act 2017 19. Sur cette question voir : E.L. O’Donnell and J. Talbot-Jones, “Creating Legal Rights for Rivers: Lessons from Australia", New Zealand, and India", préc.

20. Voir l'affaire relative au fleuve Vicabamba. Richard Frederick Wheeler et Eleanor Geer Huddlee c. Gouvernement provincial de Loja et autres, n¹1121-2011-00102.

21. High Court of Uttarakhand, Mohd Salim v State of Uttarakhand \& others, Writ Petition (PIL) No.116 of 2015, 20 mars 2017 (https://drive.google.com/file/d/ 0BzXilfcxe7yuM3VRWTZDeEtmSGc/view High); Court of Uttarakhand, Writ Petition (PIL) No.140 of 2015, 30 march 2017 (http://lobis.nic.in/ddir/uhc/RS/judgement/31-03- 017/ RS30032017WPPIL1402015.pdf). 
22. Court of Uttarakhand, Writ Petition (PIL) préc. (https://drive.google.com/file/d/ 0BzXilfcxe7yuM3VRWTZDeEtmSGc/view High); p. 63

23. Cour constitutionnelle colombienne, 10 novembre 2016, Centro de Estudios para la Justicia Sociale “Tierra Digna” , T-622 de 2016 (résumé : https://notreaffaireatous.org/wp-content/ uploads/2019/05/TierraDigna.pdf)

24. Ibid. para. 5.7. Toujours en Colombie, la Cour suprême consacre en 2018 la personnalité juridique de la forêt Amazonienne

25. N, Rühs and A; Jones, "The Implementation of Earth Jurisprudence through Substantive Constitutional Rights of Nature"n Sustainability, 8, 174.La notions de « rule of law » de la nature y est défendue à partir du « paradigme du constitutionnalisme.

26. Jusqu'à proposer une « déclaration des droits biotiques : A. Nash, “The Case for Biotic Rights", préc. p. 245.

27. Véronique Champeil-Desplats écrit: «Il reste qu'avec l'expression «droits et libertés fondamentaux ", le sujet universel des droits disparaît et ne devient qu'une présupposition et une possibilité parmi d'autres ». V. Champeil-Desplats, Théorie générale des droits et libertés, Perspective analytique, Paris, Dalloz, 2019 p. 79.

28. P. Brunet, « Vouloir pour la nature. La représentation juridique des entités naturelles », préc., p. 2. 4 : «la difficulté majeure est de parvenir à concilier la liberté des individus - obtenue de haute lutte et sur laquelle est fondée la démocratie représentative - avec des objectifs de protection, de conservation, de préservation ou encore de précaution - voire de compassion envers des objets naturels, objectifs qui supposent une certaine limitation de cette liberté ».

29. E. Millard, «Sur le caractère pré-moderne du néo-constitutionnalisme latino-américain », in O. Cayla et J.-L. Halpérin (dir.), Néo ou rétro-constitutionnalismes, Mise en perspective de la démocratie constitutionnelle contemporaine, Mare \& Martin, Paris, 2018, pp. 211-228, p. 133 : « Le symbole est fort et quand bien même il demeurerait au stade de l'énonciation, du programme politique, il accrédite une déconstruction radicale du principal sinon unique acquis de la modernité juridique : les droits sont ceux des êtres humains et ils se conçoivent à partir de chaque individualité humaine "

30. M. A. Hermitte, "La nature, sujet de droit ?", préc., p. 175 et s. Elle écrit à propos de l'approche «technique »: "Dans le cadre de cette conception technique, la qualité de sujet de droit peut être conférée en tant que de besoin, particulièrement pour tout ce qui est vivant, donc porteur de besoins propres à assurer la survie de l'individu, de la population ou de l'espèce, ce qui n'implique pas de les satisfaire tous "

31. Le caractère sacré est la plupart du temps considéré à travers les croyances des populations autochtones.

32. L'article 72 de la Constitution équatorienne est plus précis : «La nature a droit à la restauration. Cette restauration sera indépendante de l'obligation faite à toute personne d'indemniser les individus et les collectifs qui dépendent des systèmes affectés. » Enfin L'article 73 précise les obligations de l'État qui « devra prendre toute mesure de précaution et d'interdiction à l'égard des activités pouvant conduire à l'extinction d'espèces, à la destruction d'écosystèmes ou à l'altération permanente des cycles naturels. L'introduction d'organismes vivants ou inertes pouvant affecter de manière définitive le patrimoine génétique national est interdite ".

33. "Rivers, Forests, Lakes, Water Bodies, Air, Glaciers and Springs have a right to exist, persist, maintain, sustain and regenerate their own vital ecology system. The rivers are not just water bodies. These are scientifically and biologically living" High Court of Uttarakhand, Writ Petition (PIL) No.140 of 2015, 30 march 2017 préc. (https://drive.google.com/file/d/ 0BzXilfcxe7yuM3VRWTZDeEtmSGc/view High), p. 61.

34. J.A. Nash, “The Case for Biotic Rights”, préc. ,p. 243-244: “This paper strongly rejects biotic egalitarianism, arguing, in contrast, for a graded model of rights. The value-creating and value- 
experiencing capacities of humans are morally relevant differences between humans and all other species which justify preferential treatment for humans in conflict situations"

35. Une manifestation particulièrement éclatante de ce naturalisme provient de la doctrine de la «jurisprudence de la terre ». Voir M. Maliney, P. Siemen, «Responding to the great work: the role of earth jurisprudence and wild la in the 21th century »,Environmental and Earth Law Journal (EELJ): Vol. 5 : Iss. 1 , Article 3.

disponible : https://lawpublications.barry.edu/ejejj/vol5/iss1/3. "Earth jurisprudence then, is an emerging theory of law and governance that requires a radical rethinking of humanity's place in the world, to acknowledge the history and origins of the Universe as a guide and inspiration to humanity and to see our place as one of many interconnected members of the Earth community." (p. 9).

36. Jugement du 20 mars 2017 préc., para. 10.

37. La même formule est reprise dans les deux décisions" This recognition is for subserving the needs and faith of the society"

38. Dans le même sens voir M.-A. Hermitte, "Artificialisation de la nature et droit du vivant ", préc., p. 269. „C'est l'arrêt sur le Gange qui aborde le plus directement la question de la création de sujets de droit non humains, donnant un véritable cours de droit. Il déclare n'être tenu par aucune tradition réservant la catégorie à des entités humaines et s'autorise à l'ouvrir autant que nécessaire « dans l'intérêt de la société ».Elle insiste sur la présence de l'argument tenant à « la poussée des évolutions « scientifico-socio- politiques »

39. Sur cette la mise en place concrète de ces mécanismes de représentation voir $\mathrm{P}$. Brunet, "Vouloir pour la nature. La représentation juridique des entités naturelles », préc., et M.-A. Hermitte, « Artificialisation de la nature et droit du vivant », préc.

40. J.A. Nash, "The Case for Biotic Rights", préc., p. 242-244.Il rejet l'« égalitarisme biotique» (tous être vivants doivent être traités de la même manière) au profit d'un "modèle graduel des droits» privilégiant, en cas de conflit, les humains: "This paper strongly rejects biotic egalitarianism, arguing, in contrast, for a graded model of rights. The value-creating and valueexperiencing capacities of humans are morally relevant differences between humans and all other species which justify preferential treatment for humans in conflict situations »

41. Comme l'écrit M.-A. Hermitte : "Or la vision technique du sujet de droit ne dit rien du sujet, hormis sa capacité à ester en justice. Ce n'est qu'à un niveau logique second que l'on attache à ce support des attributs propres à chaque type de sujet. La personne morale n'a, à ce titre, pas les mêmes attributs que la personne physique. Si le régime juridique distinct des personnes physiques et morales est une simple construction juridique comme on le pense le plus souvent aujourd'hui, une institution résultant d'actes de volonté du souverain et non pas le résultat d'une nature humaine, alors on peut reconnaître aux non-humains la qualité de sujets de droit, même si leurs droits peuvent être profondément différents des nôtres ». M. A. Hermitte, « La nature, sujet de droit?", préc., p. 197.

42. Sur l'objection de la «rivalité » entre être humains et autres élément de la nature voir P. Brunet, «Les Droits de la nature et la personnalité juridique des entités naturelles en NouvelleZélande: un commun qui s'ignore? ", préc. p. 42 et s. : Ainsi « Rien n'empêche donc de penser des droits spécifiques pour les entités naturelles, comme pour les animaux, sans confondre les droits de ces entités avec ceux des humains. le droit contemporain fait la différence entre les droits de l'Homme ou droits fondamentaux et les autres droits ".

43. E. L. O'Donnell et J. Talbot-Jones, “Creating legal rights for rivers: lessons from Australia, New Zealand, and India", préc.

44. Pierre Brunet aborde cette question : « Les gardiens de ces entités naturelles pourraient-ils se faire aussi les «porte-parole » de ces entités ? Autrement dit, peut-on penser une forme de représentation politique de la nature ?». P. Brunet «Les Droits de la nature et la personnalité juridique des entités naturelles en Nouvelle-Zélande: un commun qui s’ignore? », préc, p. 43. 
45. Par exemple, le jugement de la Haute Cour de l'Uttakhland du 30 mars 2017 (préc.): “The past generations have handed over the 'Mother Earth' to us in its pristine glory and we are morally bound to hand over the same Mother Earth to the next generation" (p.62). Ou, concernant le jugement de la Cour constitutionnelle colombienne relative au fleuve Atrato (préc.) : "Indeed, taking into account that the environment and its biodiversity are part of the vital environment of man and that it is essential for their survival and that of future generations, our Political Charter has rightly recognized the imp", para. 5.4

46. J. Colon-Rios, "Constituent Power, the Rights of Nature, and Universal jurisdiction", in McGill Law Journal, vol. 60, n 1, 2014, p. 127-172, p. 142.

47. Véronique Champeil-Desplats écrit que les droits de la nature s'apparentent moins à « une humanisation des espèces naturelles" qu' à " une naturalisation de l'Homme ». V. ChampeilDesplats, Théorie générale des droits et libertés, op. cit., p. 81.

48. R. Eckersley, “Greening Liberal Democracy: The Rights Discourse Revisited” in Brian Doherty and Marius de Geus, Democracy and Green Political: Sustainability, Rights and Citizenship Thought, London, Routledge, 1996. Dans un sens similaire, J. Colon Rios "Constituent Power, the Rights of Nature, and Universal jurisdiction", préc.

49. Pour une approche plus générale de ces contextes: L. Cano Pecharromann, « Rights of Nature : Rivers that can stand in Court»,Resources 2018,7(1), 13; https://doi.org/10.3390/ resources7010013.

50. J. Colon-Rios et. Joel, "The Rights of Nature and the New Latin American Constitutionalism" New Zealand Journal of Public and International Law, 2015 ; Victoria University of Wellington Legal Research Paper No. 119/2017. Disponible SSRN: https://ssrn.com/abstract=2871584. A. Brites Osorio de Oliveira, «Les droits de la nature dans le nouveau constitutionnalisme latinoaméricain à partir du regard de l'anthropologie juridique », in TraHs $n^{\circ} 3$, numéro spécial «Buen vivir: balance y experiencias en los diez años de Constitución de Ecuador ",|2018, disponible : http://www.unilim.fr/trahs. Pour une approche critique de ce nouveau constitutionnalisme : E. Millard, «Sur le caractère pré-moderne du néo-constitutionnalisme latino-américain », préc.

51. Dans le même sens le Préambule de la Constitution bolivienne insiste sur le dépassement de « l'État colonial, républicain et néolibéral »; la jurisprudence colombienne se rattache aussi à ce mouvement lors qu'elle affirme, dans sa décision relative au fleuve Atrato, les principes constitutionnels de la «diversité culturelle et ethnique de la nation Cour constitutionnelle colombienne, 2016, préc., para. 5.23

52. Sur le contexte politique de la constitution équatorienne voir: A. Brites Osorio de Oliveira, «Les droits de la nature dans le nouveau constitutionnalisme latino-américain à partir du regard de l'anthropologie juridique ", préc.

53. Sur le contexte néo-zélandais voir P. Brunet, «Les Droits de la nature et la personnalité juridique des entités naturelles en Nouvelle-Zélande: un commun qui s'ignore? », préc., V. David, «La nouvelle vague des droits de la nature. La personnalité juridique reconnue aux fleuves Whanganui, Gange et Yamuna ", préc.C. Rodgers, "A new approach to protecting ecosystems: the Te Awa Tupua (Whanganui River Claims Settlement)”, Env. L. Rev., vol 19, 2017, p. 266-279.

54. En outre, la loi affirme que dépend la santé des communautés riveraines dépend de la santé des communautés riveraines. Toute décision relative à ces ensembles naturels devra être prise par un conseil composé paritairement de membres du gouvernement et de membre des communautés.

55. Cette cosmologie se traduit notamment dans la formule « Ko au te awa, ko te awa ko au" qui signifie "Je suis la rivière et la rivière est moi".

56. Véronique Champeil-Desplats a récemment donné une définition du pluralisme juridique: «le pluralisme juridique est la situation, pour un individu, dans laquelle des mécanismes juridiques relevant d'ordonnancements différents sont susceptibles de s'appliquer à cette situation » (para. 12) tout en évoquant les risques de transformations : " Reste alors à repérer les 
effets normatifs que peuvent produire ce type d'internalisation et de changement d'environnement normatif à l'égard des concepts déposés. Laisse-t-il, en l'occurrence, indemne la conception du concept de Pacha mama, ou celui-ci, intégré dans un cadre étatique et constitutionnel qui lui est initialement étranger, voit-il sa signification se transformer, le changement de format impliquant alors aussi finalement un changement de contenu normatif?»( para. 35). V. Champeil-Desplats, «Droit, pluralité des modes de normativité et internormativité. Regard juridique", in La Revue des droits de l'homme, n¹6, 2019, disponible :https://journals.openedition.org/revdh/6413.

57. Selon Victor David « Elle témoigne de la capacité grandissante du droit « moderne », lorsqu'il est coconstruit d'une manière participative avec le concours de sociétés traditionnelles à prendre compte la véritable identité des sociétés qu'il ambitionne de régir » V David, «La nouvelle vague des droits de la nature. La personnalité juridique reconnue aux fleuves Whanganui, Gange et Yamuna ", préc. , p. 423

58. En ce sens Pierre Brunet écrit à propos du cas de la Nouvelle-Zélande : « Mais peut-être est-ce escamoter la principale difficulté que tente d'ailleurs de résoudre ce même dispositif, à savoir que la cosmologie Maori n'est pas « juridique » - les croyances ne s'imposent pas en vertu d'un ensemble de règles - tant que le droit positif ne lui confère pas ce statut. Dans ces conditions, on peut y voir une solution " pragmatique ", un compromis politique,voire un mécanisme de cogestion pour neutraliser les questions de propriété tout en faisant preuve de respect », $\mathrm{P}$. Brunet, «Les Droits de la nature et la personnalité juridique des entités naturelles en NouvelleZélande: un commun qui s'ignore?», préc., p. 47. Voir aussi pour le cas latino-américain et dans le même sens Eric Millard: "Si le droit est effectivement consubstantiel d'une conception ethnocentrée, il peut difficilement en même temps être un moyen de remise en cause de cette conception et chercher à réaliser une autre cosmogonie que celle dont il dérive est un leurre, qui n'aurait d'intérêt stratégiquement que si à un moment de l'histoire les effets de sa prise en compte étaient visibles». E. Millard, «Anthropocène: Des droits naturels aux droits de la nature ", préc., p. 94.

59. Comme l'écrit A. Brites Osorio de Oliveira, «Les éléments de la culture andine insérés dans le texte constitutionnel ont pris une forme renouvelée en raison de la nécessité de les harmoniser avec d'autres idéaux contemporains, avec la reconnaissance du besoin de valoriser et de respecter les multiples formes de coexistence, le pluralisme existant en Équateur et aussi en raison de l'évolution des discussions sur l'inclusion des droits de la nature durant le processus constituant ». Elle considère ainsi que «Ces avancées constitutionnelles ne sont pas seulement le produit des manifestations et des revendications amérindiennes, elles sont aussi le résultat du travail de juristes et autres professionnels militants des droits de la nature ». Elle cite notamment le Président de l'Assemble constituante: "Acosta affirme aussi que, pour aboutir à une " démocratie de la Terre ", il est essentiel d'observer l'harmonie entre les droits de l'Homme et les droits d'autres communautés naturelles de la Terre ; de permettre aux droits des écosystèmes d'exister et de suivre leurs propres cycles vitaux ; d'octroyer de la " valeur en soi-même » de la vie qui s'exprime à travers la Nature ; et une valeur propre aux écosystèmes, indépendamment de leur utilité pour l'être humain ». A. Brites Osorio de Oliveira, «Les droits de la nature dans le nouveau constitutionnalisme latino-américain à partir du regard de l'anthropologie juridique », préc.

60. Para. 3. 8 de la loi: "Te Urewera is also prized by all New Zealanders as a place of outstanding national value and intrinsic worth; it is treasured by all for the distinctive natural values of its vast and rugged primeval forest, and for the integrity of those values; for its indigenous ecological systems and biodiversity, its historical and cultural heritage, its scientific importance, and as a place for outdoor recreation and spiritual reflection".

61. Pour le cas néao-zélandais. C. J. Iorns Magallanes, « Nature as an Ancestor: Two Examples of Legal Personality for Nature in New Zealand», Vertigo - la revue électronique en sciences de 
l'environnement [En ligne], Hors-série 22 | septembre 2015, mis en ligne le 10 septembre 2015, consulté le 21 janvier 2020. URL : http://journals.openedition.org/vertigo/16199 ; DOI : 10.4000/ vertigo.16199. Elle insiste sur la prévalence de la question politique sur celle de la nature 62. E. O'Donnell, «At the Intersection of the Sacred and the Legal: Rights for Nature in Uttarakhand, India ", Journal of Environmental Law, vol.30, n¹, p. 135-144; M. A. Hermitte, « Artificialisation de la nature et droit du vivant », préc.

63. Voir aussi la critique de P Brunet, «L'écologie des juges - la personnalité juridique des entités naturelles en Inde et en Colombie ", à paraitre chez Mare et Martin, 2020 (la notification de la page sera ajoutée dès que possible).

64. s'inspirant des travaux de Sanjay Kabir Bavikatte.

65. Décision de la Cour constitutionnelle colombienne relative au fleuve Atrato, préc., para. 9.27.

66. Ibid, para. 5.12: "In other words, biocultural rights are not new rights for ethnic communities; instead, they are a special category that unifies their rights to natural resources and culture, understanding them as integrated and interrelated "/ Il ne s'agit pas de "droit nouveau » selon la Cour mais d'une «catégorie spéciale » qui unifie ces droits à des ressources naturelles et à la culture de ces « communautés ».

67. Selon Pierre Brunet l'usage du concept des droits bioculturels s'explique par le fait que «la cour semble manquer d'une cosmologie propre aux populations du Chocó que le droit positif colombien pourrait traduire ou incorporer, comme dans le cas de la Nouvelle-Zélande, afin de prendre en compte la relation spécifique que ces populations entretiennent avec le fleuve. stratégique». P. Brunet, «L'écologie des juges - la personnalité juridique des entités naturelles en Inde et en Colombie ", à paraître chez Mare et Martin, 2020 (la notification de la page sera ajoutée dès que possible).

68. F. Girard, «Communs et droits fondamentaux: la catégorie naissante des droits bioculturels ", « Le droit des libertés en question(s), colloque $n^{\circ} 2$ de la RDLF, RDLF, chron. ${ }^{\circ} 28$, 2019, disponible http://www.revuedlf.com/droit-fondamentaux/communs-et-droitsfondamentaux-la-categorie-naissante-des-droits-bioculturels/\#_ftnref216

69. Comme l'écrit F. Girard, les populations autochtones seraient "protégées non en tant que tels, mais en tant que leurs activités et leurs valeurs sont instrumentalement liées à la protection de l'environnement »

70. Ce risque « d'atomisation et de dépolitisation du sujet de droit » est présenté par Véronique Champeil-Desplats comme inhérent à la rhétorique des droits fondamentaux, V. ChampeilDesplats, Théorie générale des droits et libertés, op. cit., p. 80.

71. Victor David évoque cite l'article 110-346 du Code de l'environnement de la Province des Iles Loyauté de Nouvelle-Calédonie est ainsi rédigé : " Le principe unitaire de vie qui signifie que l'homme appartient à l'environnement naturel qui l'entoure et conçoit son identité dans les éléments de cet environnement naturel constitue le principe fondateur de la société kanak. Afin de tenir compte de cette conception de la vie et de l'organisation sociale kanak, certains éléments de la Nature pourront se voir reconnaitre une personnalité juridique dotée de droits qui leur sont propres, sous réserve des dispositions législatives et réglementaires en vigueur ", Délibération $n^{\circ}$ 2016-13/API du 6 avril 2016 portant adoption du Code de l'environnement de la province des îles Loyauté, Journal officiel de la Nouvelle-Calédonie, 23 juin 2016, p. 5941.V. David, « La nouvelle vague des droits de la nature. La personnalité juridique reconnue aux fleuves Whanganui, Gange et Yamuna ", préc. p. 424.

72. Avec l'impulsion d'Olivier Clerc (colloque, table ronde), la question de l'adoption d'une Charte de la nature est en cours de réflexion : la collectivité de Corse a demandé une étude sur le sujet. 


\section{RÉSUMÉS}

Tous les partisans des droits de la nature partagent une critique virulente de l'anthropocène, qui renvoie à une ère de domination par l'homme de son environnement naturel. La reconnaissance des droits à la nature serait une manière de modifier nos représentations du monde (entre holisme et individualisme). Le glissement de concepts que suggère cette reconnaissance soulève donc des craintes légitimes : comment la nature pourrait-elle se substituer à l'individualisme libéral ? Certains systèmes juridiques (en Equateur, en Bolivie, en Colombie, en Inde, en NouvelleZélande) ont déjà attribué de tels droits à des fleuves, rivières, glaciers, forêts, et autres ensembles naturels en empruntant le langage du constitutionnalisme. Cette étude se focalise sur l'argumentation utilisée dans ces différents cas concrets en distinguant les registres axiologiques (la reconnaissance de droits) et politiques (la référence, même lointaine, au(x) «peuple(s) ».)

All proponents of nature rights share a deep criticism of the Anthropocene, which relates to an era of human domination over its natural environment. The recognition of the rights of nature would be a way to modify our representations of the world (between holism and individualism). The conceptual shift suggested raises legitimate fears: how could nature go beyond the liberal individualism? Some legal systems (in Ecuador, in Bolivia, in Columbia, in India, in New Zealand) have already enshrined such rights for rivers, glaciers, forests, natural areas while borrowing the language of constitutionalism. This study focuses on the different types of argumentative registers that led to this recognition by distinguishing between the axiological one (recognition of rights) and the political one (the reference, even distant, to "people").

\section{INDEX}

Keywords : rights of nature, anthropocene, constitutionalism, future generations, indigeneous people

Mots-clés : droit de la nature, anthropocène, constitutionnalisme, droit naturel, générations futures, peuples autochtones

\section{AUTEUR}

\section{MANON ALTWEGG-BOUSSAC}

Manon Altwegg-Boussac est Professeure à l'Université Paris-Est Créteil 